\title{
Research on the Design of Interface in the Micro-Portal Web Game
}

\author{
Yandong Chen, Xiuming Sun \\ Zhangjiakou Univrsty, Zhangjiakou Hebei, China \\ cyd200306@sina.com
}

Keywords: Micro-portal web game, Interface design, Attractiveness, Search efficiency

\begin{abstract}
With the development of our society, the progress of science and technology, as well as the highlight of user time's fragmentation characteristics, the characteristics of users in game industry have taken dramatically changed, and as the number of web game users has gradually increased, many companies are also paying much more attention to the development and promotion of web game. As a main way to attributing users, the design of interface in Micro-portal web game is very important. This paper studied the user's attractiveness and search efficiency by two experiments on interface layout of micro-portal web game, the picture size (or number) and with/without JS effects of micro-portal web game. This paper provides a good basis and guidance for subsequent design of interface in micro-portal web game.
\end{abstract}

\section{Introduction}

With the development of society, great progress of computer technology, as well as the highlight of user time fragmentation characteristic, game industry has undergoing taken dramatic changes. At present, web game, with lower development costs and shorter development cycles, makes many companies paying more and more attention to it. According to the report of IResearch-"Chinese web game industry Report 2010 - 2011", scale of web game market in our country was 4.81 billion in 2011, having increased $44.9 \%$, compared with it in 2010; at the same time, the populations of web game user is 46 million in 2011, having risen 24.3\% compared to 2010. Now the main web game companies includes TECENT, SOGOU, BAIDU, 4399,51 wan, 37wan, etc [1]; the number of web game increased greatly, for example, its number being 183 in 2009 and reaching a number of 997 in 2011.

The interface design of website entrance is very important for improving the website's visits, and the interface design is no longer just a simple artistic appearance and presentation of information with the popular of emotional design [2] in product design, but natural interaction of human-computer and good user experience have become a guiding design principle. In order to design a beautiful and attractive interface, the design of modern interface is based on a multi-disciplinary background, which contains ergonomics, cognitive psychology, aesthetics, interaction design, etc; further, making users enjoying good experience about the interface they browse, at the same time, attracting much more attention of them [3].

As one of entrances to website, micro-portal [4] is based on the existing website portal, and presenting the best quality content, which is integrated, to users, achieving the goal of attracting more users and improving site visits; besides, micro-portal game interface is an important mean for game companies to attract users. However, nowadays the design of interface in micro-portal web game is different from each other, having no unified style, this undoubtedly gives user very bad experience on the time they browse in micro-portal web game. Generally, micro-portal includes two forms: PC-side and mobile terminal. Currently, the main gateway to Internet industry has its own micro-portal sites, such as SOHU, SINA, NETEASE, TECENT.

It is very important to study the interface design of micro-portal, which is one of entrances to website, for improving site's visits [5] and attracting more users. Based on this background, this paper designs two experiments to study the impact of interface design in micro-portal web game on attractiveness to user and search efficiency, by designing kinds of interface. This two experiments studied the impact of interface design in micro-portal web game on attractiveness to user and search efficiency main from three aspects, including element layout of interface in micro-portal web game, 
image size(or quantity), with or without JS effect, and found that entrance in design of interface had no significant difference on users' hits and attractiveness, namely, entrance of site had no significant impact on user' behavior; the design with image on right and text on left is much more attractive than design with image on left and text on right; user stayed much longer in design with JS effect than without JS effect, meaning that design with JS effect is much more attractive than design without JS effect; users' searching efficiency improved with the increase of image size, but the hits and user's retention time having no changes over image size. This paper fills the gaps of interface design in micro-portal web game and provides a good basis and guidance for subsequent design of interface in micro-portal web game.

\section{Experiment Design}

Experiment preparation. In this paper, the experiment material was used by JSP + Mysql technology[14], and add the page to completing the hits' statistics by adding relevant statistics point in the webpage, the key code is:

// Add specific value to button

$<$ atarget="_blank"

pbflag="area_logo" $><\mathrm{i}></ \mathrm{i}></ \mathrm{a}>$

href="http://wan.sogou.com/"

class="toWan"

//add the statics script

<script type="text/javascript">

var spb_vars $=\{$

productid: "test_left_8",

ptype: "gaoyujun",

pcode: "page_left_8_JS"

\}

$<$ script $>$

<script type="text/javascript" src="http://pb.i.sogou.com/u/pb/pb.990992.js" ></script>

After the page being finished, install http debugging tools fiddler, which is a http debugging tool, we can use it capturing the user's requests by it[15].

\section{Experiment I.}

A. The purpose of the experiment.This experiment is designed to study the influence of website entrance position, different interface elements layout, image size (number) and with / without JS on webpage hits and user's residence time on the webpage.

B. Experimental method. 1) Subjects. 30 subjects contains 8 females and 22 males, all ageing from 24 to 26 years old, having the same cognitive level and experience of playing games. All subjects are right-handed , over three years' experience on Internet and visual acuity or corrected visual acuity reaching to 1.0 or more. 2) Materials. Materials are based on the JSP pages in this experiment, which is finished before produced from the JSP pages, and in order to avoid influence from color and content on operational tasks, all pages have been made of the same materials with same color, images and text content.

Six pages is needed in this experiment, the first four pages with eight images, which contains kinds of situations, that is website entrance with its position in upper left, lower left, upper right, lower right, as well as interface elements layout with images on right \& text on left, and text on right \& images on left; the last two pages with 12 images, having the same content, but one of them having no JS effect on page.

3) Experimental equipment. PC laptop computer, scrap paper, pen

4) Design for experimental variables

Independent variable: Website entrance position: top, bottom, left, right; two kinds of interface elements layout: images on left/text on right, images on right/text on left; image size (number); with/without JS effect (if we have not indicated one for them being without-JS effect, it is default with JS effect). 
The dependent variable: Hits of website entrance, hits of whole web page, subjects' residence time on the web page. Controlled variables: Text layout of interface, the use of color, user's familiarity with material content. Evaluation: Hits of website entrance, hits of whole web page, subjects' residence time on the web page

5) Subjective evaluation. Make user rating for different design through Likert7 subscales, to survey subjects' satisfaction.

C. Experimental procedure. 1) At the beginning, let the participants browse the experiment materials for 2 minutes and obtain the familiarity in some degree; 2) The experimenter tells subjects the operations carried during the experiment and show the experiment materials to them in order to let them having a certain degree of understanding about the experiment; 3) Subjects visit the pages presented to them and click the interesting content, and Each of subjects will finish the operation on six pages; 4) After completion of task, subjects give their subjective assessment and score for each design;

D. Evaluation. Data which need to be recorded contains objective data during the experiment and users' satisfaction data. Objective data are time used to search the target and number of operation mistakes during the searching process by subjects.

Considered when you click on any area on page as one hits, after subjects entering page; and consider time that subjects began to enter the current page from time to time so far away from the page as the residence time on page. The words that appear in the experimental material all have clear meaning, therefore there have no misuse during subjects' task, so that there is no need to analyze the rate of mistakes.

\section{Experiment II}

A. The purpose of the experiment

This experiment is designed to study whether interface elements layout and image size (number) have impact on the user's searching efficiency or not.

B. Experimental method

1) were tested. 30 subjects contains 8 females and 22 males, all ageing from 24 to 26 years old, having the same cognitive level and experience of playing games. All subjects are right-handed, over three years' experience on Internet and visual acuity or corrected visual acuity reaching to 1.0 or more.

2) Experimental equipment. PC laptop computer, scrap paper, pen

3) Design for experimental variables. Independent variable: Two kinds of interface elements layout: images on left/text on right, images on right/text on left; image size (number):with 8 images, 12 images. Dependent variable:Time subjects using to finish searching task, the number of operation mistakes during the searching process. Controlled variables: Text layout of interface, the use of color, user's familiarity with material content. Evaluation: Time subjects using to finish searching task, the number of operation mistakes during the searching process.

4) Subjective evaluation. Make user rating for different design through Likert7 subscales, to survey subjects' satisfaction.

C. Experimental procedure. 1) At the beginning, let the participants browse the experiment materials for 2 minutes and obtain the familiarity in some degree; 2) The experimenter tells subjects the operations carried during the experiment and show the experiment materials to them in order to let them having a certain degree of understanding about the experiment; 3) Let subjects enter the website to find the games promoted on the page and click them; Each of subjects needs to make 18 times of search operation of three pages with 3 games on each page; 4) The experimenter records the experimental data.

D. Evaluation. Data which need to be recorded contains objective data during the experiment and Users' satisfaction data. Objective data are time used to search the target and number of operation mistakes during the searching process by subjects.

The time from subjects entering page to complete 3 times searching task is regarded as operation time. Operations to other contents except target are regarded as operation mistakes. 
data.

E. Results and analysis. Export the completed data, and use statistical software SPSS15 to analysis

Table1 Paired samples T-test on the data in table1

\begin{tabular}{|c|c|c|c|c|c|c|c|c|c|}
\hline \multicolumn{10}{|c|}{ Paired Samples Test } \\
\hline & & \multicolumn{5}{|c|}{ Paired Differences } & \multirow{3}{*}{$\mathrm{t}$} & \multirow{3}{*}{ df } & \multirow{3}{*}{$\begin{array}{c}\text { Sig. } \\
\text { (2-tailed) }\end{array}$} \\
\hline & & \multirow[t]{2}{*}{ Mean } & \multirow{2}{*}{$\begin{array}{c}\text { Std. } \\
\text { Deviation }\end{array}$} & \multirow{2}{*}{$\begin{array}{l}\text { Std. Error } \\
\text { Mean }\end{array}$} & \multicolumn{2}{|c|}{$\begin{array}{l}\text { 95\% Confidence Interval } \\
\text { of the Difference }\end{array}$} & & & \\
\hline & & & & & Lower & Upper & & & \\
\hline $\begin{array}{c}\text { Pair } \\
1\end{array}$ & $\begin{array}{c}\text { With } 8 \text { img on } \\
\text { right and text on } \\
\text { left/with } 12 \mathrm{img} \\
\text { on right and text } \\
\text { on left }\end{array}$ & -3.56667 & 1.33089 & .24299 & -4.06363 & -3.06970 & -14.678 & 29 & .000 \\
\hline $\begin{array}{l}\text { Pair } \\
2\end{array}$ & $\begin{array}{c}\text { With } 12 \text { img on } \\
\text { left and text on } \\
\text { right/with } 12 \text { img } \\
\text { on right and text } \\
\text { on left }\end{array}$ & .03333 & 2.32651 & .42476 & -.83540 & .90207 & .078 & 29 & .938 \\
\hline
\end{tabular}

Firstly, the rate of mistakes in this experiment just is $1.11 \%$, which does not influence the overall results, so we will not make analysis for those mistakes.

Then, from the data in table2, we can see that the $\mathrm{P}$ value is less than 0.05 in the first group, indicating that image size has significant impact on times subjects accomplishing searching task, and more efficient for subjects accomplishing task with 8 images on the interface than with 12 images. However, $\mathrm{P}$ value is higher than 0.05 , meaning that interface elements layout has no significant impact on subjects' searching efficiency.

\section{Conclusion}

If you follow the "checklist" your paper will conform to the requirements of the publisher and facilitate a problem-free publication process.

The following conclusions can be draw by the two experiments in this paper:

1. Firstly, interface elements layout of micro-portal game has great impact on hits and attraction for user. According to the experimental data in this paper, the effect when interface elements layout use layout with images on left and text on right, is much better than that with images on right and text on left, which is in line with people's cognitive behavioral habits; Therefore, images should be placed in the left area in the interface design of micro-portal game.

2. Then, the use of JS effects in interface elements design also has significant impact on user's behavior. Thus, the main interface elements, such as images or key content, are recommended to be added JS effects in the interface design of micro-portal game, in order to make users paying more attention to them, further improve website hits.

3. Last but not the least, image size of the experiments in this paper, has significant impact on users' searching efficiency, but has no significant impact on hits and residence time on page. In order to improve users' searching efficiency in the interface design of micro-portal game, images with larger size are recommended to use, just like using eight images in this page.

4. Last, because the hits of website entrance is very little in every position, so the position of website entrance has little or none impact on user's behavior. Therefore, the position of website can be placed by some other element more attractive.

\section{References}

[1] J. van der Geer, J.A.J. Hanraads, R.A. Lupton, The art of writing a scientific article, J. Sci. Commun. 163 (2000) 51-59. 
[2] Zhentao Zhu. Research on interface design of network game, http://www.lunwentianxia.com/ product. sf.338478.1.

[3] Donald A N. Emotional design [M]. Beijing: electronicindustry press, 2005.

[4] Yanping Wu. Research of User-centered Web Design [D]. Hangzhou: Zhejiang university, 2011.

[5] Yuan Yi, Lin Sheng. Introduction to the website design of government portal [J], computer network. October 2012.

[6] Suiming Qin, Dongmei Ma. Analysis and study of web interface design. Shanxi university of science and technology, packaging engineering. April 2013

[7] wei Liu, Xiugan Yuan etc. Comprehensive evaluation index and evaluation method to the fitment between man-machine interface display and control [J]. Chinese journal of safetyscience, pp. 32-35. April 2004.

[8] lan Lan, gang Zhao, na Li. The analysis of visual components based on web interface [J]. Journal of art and design, pp. 31-33, February 2012.

[9] Haibo Lin, wen Lai. How to use web2.0 technology to improve the user experience [J]. Journal of library science research, pp. 17-19, December 2008.

[10] Qiongyao Ren. Research on culture elements in the form of life precious based on the user's web [D]. Hangzhou: zhejiang university, 2012

[11] Haiyan Huang, Hui Zhang. Web and graphic design [J].Journal of packaging engineering, vol. 1, pp. 126-127, 2004.

[12] Su Zhou etc. The man-machine interface design, science press, 2007.

[13] Theo Mandel, The Elements of The User Interface Design,New York: Wiley Computer Publishing, 1997.

[14] Zhuangjia Li. Modern color design [M]. Beijing: light industry press, 1987.

[15] Hanming Tang. Simple introduction to the development,optimization and management maintenance of MYSQL database[M]. People's posts and telecommunications publishing house, March 2010

[16] Hua Zou etc. The design and examples of Servlet/JSP program. Beijing: people's posts and telecommunications publishing house, 2014.

[17] Wei Xue. Analysis and application SPSS statistical method[M]. Beijing electronic industry publishing house, 2013.

[18] Wenshuan Ling, Liluo Fang. Measures of psychologic and behavior[M]. Beijing: mechanical industry publishing house, 2013.

[19] Jesse J G. The Elements of User Exper- ience: User-Centered Design for the Web (Vo ices That Matter) [M]. Beijing: mechanical industry press, 2007. 